\title{
Phenotypic plasticity influences the eco-evolutionary dynamics of a predator-prey system
}

\author{
Beat B. Fischer, ${ }^{1}$ Marek Kwiatkowski,${ }^{2,3}$ Martin Ackermann, ${ }^{4,5}$ Jasmin Krismer, ${ }^{1}$ Severin Roffler,${ }^{1}$ \\ Marc J. F. Suter, ${ }^{1}$ Rik I. L. Eggen, ${ }^{1}$ and Blake Matthews ${ }^{6,7}$ \\ ${ }^{1}$ Eawag, Swiss Federal Institute of Aquatic Science and Technology, Department of Environmental Toxicology, \\ Duebendorf, Switzerland \\ ${ }^{2}$ Eawag, Swiss Federal Institute of Aquatic Science and Technology, Department of Aquatic Ecology, Duebendorf, Switzerland \\ ${ }^{3}$ Institute of Biology, University of Neuchâtel, Switzerland \\ ${ }^{4}$ Eawag, Swiss Federal Institute of Aquatic Science and Technology, Department of Environmental Microbiology, \\ Duebendorf, Switzerland \\ ${ }^{5}$ Department of Environmental Systems Science, ETH Zurich, Switzerland \\ ${ }^{6}$ Eawag, Swiss Federal Institute of Aquatic Science and Technology, Department of Aquatic Ecology, Center for Ecology, \\ Evolution and Biogeochemistry, Kastanienbaum, Switzerland
}

\begin{abstract}
There is increasing evidence that rapid phenotypic evolution can strongly influence population dynamics, but how are such eco-evolutionary dynamics influenced by the source of trait variation (i.e., genetic variation or phenotypic plasticity)? To investigate this, we used rotifer-algae microcosm experiments to test how the phenotypic and genetic composition of prey populations affect predator-prey population dynamics. We chose four genetically distinct strains of the green alga Chlamydomonas reinhardtii that varied in their growth rate, standing levels of defense, and inducible defense. To additionally test for strain specificity of plasticity responses, we quantified protein expression of each strain in the presence and absence of rotifer predators (Brachionus calyciflorus). We then tested how different strain combinations influenced the outcome of pairwise competition trials with and without rotifer predation. We tracked individual strain frequencies using quantitative polymerase chain reaction ( $\mathrm{qPCR}$ ), and compared the observed dynamics to a suite of ecoevolutionary models of varying complexity. We found that variation in trade-offs between growth and defense between algal strains strongly influenced the outcome of competition and the overall predator-prey dynamics. Our purely ecological model of the observed dynamics, which allowed for the presence of phenotypic plasticity but no trait variation between strains, never outperformed any of our eco-evolutionary models in which strains could have different trait values. Our best fitting eco-evolutionary model allowed strains to differ in an inducible defense trait. Overall, our results provide some of the first experimental evidence that variation in phenotypically plastic responses among prey genotypes can be an important component of eco-evolutionary dynamics in a predator-prey system.
\end{abstract}

Key words: coexistence; competition; eco-evolutionary dynamics and feedbacks; induced defense; intrapopulation diversity; phenotypic plasticity; predator-prey systems; proteomics; rapid evolution; tradeoffs.

\section{INTRODUCTION}

A central goal of ecology and evolution is to predict how the phenotypic and genetic variation among individuals influences population and community dynamics (Kokko and López-Sepulcre 2007, Ezard et al. 2009, Ozgul et al. 2009, 2010, Hanski 2012, Reznick 2013, Smallegange and Coulson 2013). There is increasing evidence that intrapopulation phenotypic and genetic variation of certain species (e.g., top predators, founder species, keystone species) can have wide-ranging effects on both the structure (Crutsinger et al. 2006, Harmon et al. 2009, Matthews et al. 2011, Bassar et al. 2012, Whitham et

Manuscript received 24 January 2014; revised 1 May 2014; accepted 8 May 2014. Corresponding Editor: E. Van Donk.

${ }^{7}$ Corresponding author. E-mail: blake.matthews@eawag.ch al. 2012, Agrawal et al. 2013) and dynamics (Hairston et al. 2005, Pelletier et al. 2007, Keith et al. 2010, Ellner et al. 2011, Becks et al. 2012) of communities. There is also a growing recognition that phenotypic evolution and community dynamics can occur on similar timescales (Thompson 1998, Hairston et al. 2005, Fussmann et al. 2007, Cortez 2011, Schoener 2011), leading to a contemporary interplay between evolutionary and ecological dynamics in nature (Post and Palkovacs 2009, Matthews et al. 2011, Schoener 2011). While recent research on ecoevolutionary research has documented the pervasive influence of trait variation on communities, it is an ongoing challenge to determine how the origins of trait variation (e.g., plasticity, genetic variation) influence population, community, and ecosystem dynamics (Bolnick et al. 2011, Ellner et al. 2011, Farkas et al. 2013). 
Intrapopulation variation in functional traits can arise from a combination of both genetic diversity and phenotypic plasticity, and distinguishing between these sources is important for predicting the effects of trait variation on population and community dynamics (Bolnick et al. 2011, Cortez 2011, Schreiber et al. 2011, Yamamichi et al. 2011, Kovach-Orr and Fussmann 2012). For example, variation in prey defense traits originating from plasticity vs. genetic variation can have contrasting effects on the cyclical dynamics (Cortez 2011) and the persistence and stability of predator-prey communities (Kovach-Orr and Fussmann 2012). The origin of trait variation is also important for understanding the dynamics of competitive communities, because variation in the heritability of traits involved in inter- and intraspecific competition can influence the likelihood of species coexistence by affecting the relative strength of natural selection and the speed of adaptation (Vasseur et al. 2011). In some situations, the rapid phenotypic response of plastic traits is analogous to rapid evolution of traits with high heritability (Vasseur et al. 2011), but evolving systems can also exhibit certain cyclical dynamics that are not possible in systems with adaptive phenotypic plasticity but no evolutionary change (Cortez 2011). Although recent empirical studies also suggest an important distinction between the effects of heritable and non-heritable trait change on population and community dynamics (Ozgul et al. 2010, 2009, Ellner et al. 2011), many eco-evolutionary experiments primarily focus on how heritable trait variation affects population and community dynamics (Becks et al. 2010, Farkas et al. 2013). Hence, there is a growing need for experimental tests of how trait variation originating from multiple sources (e.g., plasticity and genetic variation) can influence eco-evolutionary dynamics (Bolnick et al. 2011, Hanski 2012).

Studying the eco-evolutionary dynamics of predatorprey systems is a useful starting point for testing how the origins of trait variation might influence population and community dynamics (Fussmann et al. 2000, Yoshida et al. 2003, 2004, 2007, Becks et al. 2010, 2012). In prey populations, plasticity-induced changes in defense traits can occur readily within a generation and might lead to rapid increases in individual fitness without changes to the genotype distribution, whereas natural selection can drive genetic changes over multiple generations that lead to more persistent trait change within populations. Such differences in timescales are known to affect the stability and dynamics of predator-prey models (Cortez 2011, Schreiber et al. 2011), where adaptive phenotypic plasticity tends to synchronize predator-prey oscillations and increase system stability (Yamaguchi et al. 2011, Kovach-Orr and Fussmann 2012) and rapid evolution of prey traits tends to desynchronize cycles and increase the lag between predator and prey oscillations (Cortez 2011). In models that include prey evolution the predicted increase in lag matches qualitatively with empirical work on the predator-prey dynamics of rotifers and algae in microcosm experiments (Yoshida et al. 2004, Becks et al. 2010, 2012). In such experiments, the evolution of algal defense traits can cause predator-prey cycles to shift from a quarterphase to a half-phase lag (Yoshida et al. 2003, 2004), which is an effect that is unlikely caused by the mere presence of phenotypic plasticity in algal defense traits (Cortez 2011). However, algal species and strains can also differ in the reaction norms of prey defense traits, and there is considerable experimental work in some species showing how plastic strains can increase population stability of upper trophic levels (Verschoor et al. 2004, Van der Stap et al. 2006, 2007). However, less is known about how the dynamics of intrapopulation variation in prey defense traits, arising from the combination of both genetic and plasticity differences among strains, can affect the overall system dynamics.

In previous eco-evolutionary experiments with rotifers and algae (Chlamydomonas reinhardtii), prey evolution was shown to occur via changes in the proportions of genetically distinct strains over time, starting from experimentally manipulated levels of standing genetic variation (Becks et al. 2010, 2012). Among the prey strains, there is presumed to be genetic variation underlying the traits governing the trade-off between growth rate and defense, such that at high rotifer density grazing pressure causes evolution toward better-defended but slower-growing strains, whereas at low rotifer density competition for limiting nutrients causes evolution towards poorly defended but faster-growing strains (Yoshida et al. 2007, Becks et al. 2012). An important evolving trait in these algal populations of Chlamydomonas reinhardtii is the propensity of cells to clump into palmella, which are aggregates of multiple cells that have lost their flagella and are enveloped in a mucous envelope. The palmelloid phenotype is thought to provide resistance to both ingestion and digestion by rotifers (Lurling and Beekman 2006, Van der Stap et al. 2009). Direct measurements of broad-sense heritability of palmella formation in Chlamydomonas suggest that this defense trait can be highly heritable in some strains (Becks et al. 2010). In addition, contrasting patterns of gene expression in a Chlamydomonas population (multiple strains) at different stages of the predator-prey cycle suggest that multiple gene transcription pathways might lead to a defended algal phenotype (Becks et al. 2012). However, there is also some evidence for the induction of Chlamydomonas defenses in response to rotifer predation (Lurling and Beekman 2006), as is common in other algal species (Van der Stap et al. 2006, Van der Stap al. 2009), and strain specificity in the phenotypic response of Chlamydomonas to multiple environmental stressors, including rotifer predation (Fischer et al. 2012). Taken together, such previous work suggests that a rotifer-Chlamydomonas model system is useful for experimentally testing how intrapopulation variation in the reaction norms of prey 
TABLE 1. Parameter estimates (mean $\pm \mathrm{SE}$ ) based on 48-h incubation experiments for each algal strain of C. reinhardtii, where standing and total protection are calculated from the fraction of cells in palmella without and with rotifers, respectively.

\begin{tabular}{lcccccc}
\hline \hline & & & & & \multicolumn{2}{c}{ Heritability } \\
\cline { 3 - 7 } \multicolumn{1}{c}{ Parameters } & CC125 & CC2342 & CC2343 & CC2344 & $H^{2}$ & Slope \\
\hline Growth rates, $q$ (cells/d) & $1.14^{\mathrm{a}} \pm 0.05$ & $1.54^{\mathrm{b}} \pm 0.12$ & $1.48^{\mathrm{ab}} \pm 0.09$ & $1.57^{\mathrm{b}} \pm 0.12$ & 0.85 & 0.99 \\
Standing protection $(s)$ & $0.10^{\mathrm{a}} \pm 0.03$ & $0.02^{\mathrm{a}} \pm 0.01$ & $0.05^{\mathrm{a}} \pm 0.04$ & $0.43^{\mathrm{b}} \pm 0.11$ & 0.80 & 0.98 \\
Induced protection $(i)$ & $0.13^{\mathrm{a}} \pm 0.06$ & $0.09^{\mathrm{a}} \pm 0.04$ & $0.50^{\mathrm{b}} \pm 0.10$ & $0.14^{\mathrm{a}} \pm 0.03$ & 0.50 & 0.95 \\
Total protection $(T)$ & $0.31^{\mathrm{ab}} \pm 0.11$ & $0.20^{\mathrm{a}} \pm 0.07$ & $0.62^{\mathrm{b}} \pm 0.08$ & $0.56^{\mathrm{ab}} \pm 0.10$ & 0.65 & 1.0 \\
\hline
\end{tabular}

Notes: Induced protection is calculated as described in Materials and methods: Strains and growth conditions. Broad-sense heritability $\left(H^{2}\right.$ and the slope of the relationship between the trait measured in the parental generation $[x$-axis] and in a subsequent generation [ $y$-axis]) is calculated following Becks et al. (2010). Significant differences between strains $(P<0.05)$ are indicated with superscripted lowercase letters based on Tukey's post hoc test, following an ANOVA (means sharing a letter are not significantly different).

defense traits might affect eco-evolutionary dynamics between predators and their prey.

Here, we experimentally tested how the phenotypic and genetic variation among strains of an algal prey population (Chlamydomonas reinhardtii) can influence the dynamics of a predator-prey system (one rotifer species, two algal strains). To do this, we used laboratory microcosm experiments with a rotifer predator (Brachionus calyciflorus) and a collection of genetically different algal strains (Chlamydomonas reinhardtii) that have quantifiable differences in both growth rate and standing levels of defense (fixed traits), as well as in the reaction norm of one induced defense trait (plastic trait). To test whether rotifers induce rapid and functionally significant phenotypic responses in our algal strains, we measured protein expression of each strain in the presence and absence of rotifers, and assessed variation in expression across multiple functionally relevant groups of proteins (e.g., cell wall structure, metabolism, energy conversion). We then manipulated the starting phenotypic and genetic composition of the algal community so as to experimentally test how different trait combinations between pairs of strains affect overall predator-prey community dynamics. We developed a quantitative polymerase chain reaction (qPCR) method that allowed us to track the frequency of the two algal strains over the course of each experiment. We then determined which trait differences between competing algal strains was the most important for explaining the observed community dynamics by quantitatively comparing the outcome of our experiments to a range of eco-evolutionary models of varying complexity. Overall, this allowed us to experimentally test how variation in the origin of functional trait variation within prey populations (i.e., genetic or plastic) influences overall predator-prey dynamics.

\section{Materials And Methods}

Strains and growth conditions.-In a previous study, we documented differences between eight genetically distinct strains of Chlamydomonas reinhardtii in terms of their growth rate and protection against rotifer predation (Fischer et al. 2012). For the current study, we selected two unpalatable strains (CC2343, CC2344) and two palatable strains (CC125, CC2342), based on previous feeding experiments (Fischer et al. 2012), and quantified variation in three ecologically relevant traits, including growth rate, standing protection (fraction of cells clumped in palmella), and induced defense (induction of the palmelloid phenotype in the presence of rotifers; Table 1). The four strains (CC125, CC2342, CC2343, and CC2344 from the Chlamydomonas Resource Center, St. Paul, Minnesota, USA) and a clonal population of the rotifer Brachionus calyciflorus Pallas (Florida Aqua Farms, Dade City, Florida, USA) were cultured in Talaquil media as described in Fischer et al. (2012). To measure growth rates of the individual algal strains we incubated them in $100 \mathrm{~mL}$ of fresh Talaquil media at a concentration of $2 \times 10^{5}$ cells $/ \mathrm{mL}$. Algal growth was determined by measuring chlorophyll fluorescence at 670-690 nm (excitation at 436-444 $\mathrm{nm})$ at 0,24 , and $48 \mathrm{~h}$.

To measure standing and induced levels of defense we used a Leica DM IL inverted contrasting microscope (Leica, Wetzlar, Germany) to determine the proportion of total cells in palmella after a 48 -h incubation either without rotifers (standing protection) or with rotifers (induced defense). Standing protection $(s)$ is the proportion of cells that are in palmella of at least two cells compared to all cells present in the culture in the absence of rotifers. Total protection $(T)$ is measured in the same way but in the presence of rotifers. Induced protection $(i)$ is calculated using the equation $(1-T)=$ $(1-s) \times(R / g)^{-i}$, where $g$ is a threshold rotifer density that initiates an induced defense (Table 2), and $R$ is the abundance of rotifers. This is a similar formulation used in two previous models of plasticity of algal defenses (Vos et al. 2004, Yamamichi et al. 2011). We assumed an upper limit of 2 for $i$, given a maximum rotifer density of 50 individuals/ $\mathrm{L}$ and a maximal increase in the proportion of cells in palmella from $s=0$ to $t=0.99$. We have chosen this simple model because it assumes a fixed threshold rotifer density for defense induction and has a single free parameter to characterize variation in induced defenses (Yamamichi et al. 2011); however, it is also possible that strains vary in their threshold $(g)$ or exhibit different and more complex relationships between rotifer density and total protection. 
TABLE 2. Parameter estimates for the differential equations used to model predator-prey dynamics.

\begin{tabular}{cclll}
\hline \hline Parameter & Value & \multicolumn{1}{c}{ Unit } & \multicolumn{1}{c}{ Description } & Reference \\
\hline$\beta_{\mathrm{B}}$ & 2.25 & $\mathrm{~d}^{-1}$ & rotifer recruitment per capita & this study \\
$\chi_{\mathrm{B}}$ & 125.0 & no. rotifers/million algae cells & $\begin{array}{l}\text { rotifer conversion } \\
\text { density for response switch }\end{array}$ & this study \\
$Q^{*}$ & 0.05 & million algae cells $/ \mathrm{mL}$ & Becks et al. (2010) \\
$K_{\mathrm{B}}$ & 0.15 & million algae cells $/ \mathrm{mL}$ & density for rotifer half-saturation & Becks et al. (2010) \\
$k$ & 0.8 & million algae cells $/ \mathrm{mL}$ & prey carrying capacity & this study \\
$g$ & 5.0 & no. rotifers $/ \mathrm{mL}$ & induced defense threshold & this study \\
$m$ & 0.055 & $\mathrm{~d}^{-1}$ & rotifer mortality rate & Becks et al. (2010) \\
$\delta$ & 0.69 & $\mathrm{~d}^{-1}$ & dilution rate & this study \\
$s_{0}, s_{1}$ & in $[0,1]$ & dimensionless & degree of standing protection & this study \\
$q_{0}, q_{1}$ & in $[0.72,3.6]$ & cells $/$ d & intrinsic algal growth rate & this study \\
$i_{0}, i_{1}$ & in $[0,2]$ & dimensionless & this study & this of induced protection \\
\hline
\end{tabular}

Note: Subscript B in $\beta_{\mathrm{B}}, \chi_{\mathrm{B}}$, and $K_{\mathrm{B}}$ refers to breeding rotifers.

Protein expression analyses.-We used proteomics (Martínez-Fernández et al. 2010, Silvestre et al. 2012) to test whether our four algal strains that vary in important functional traits (Table 1) also have different protein expression profiles (i.e., a proteomic phenotype) in the absence of predation, and whether rotifers induce a significant change in protein expression consistent with a rapidly induced phenotypic response to rotifer predation (i.e., within $48 \mathrm{~h}$ ). To test this, we incubated individual algal strains with and without rotifers, separated the rotifers by filtration, washed the algal cells twice with sterile water, froze them in liquid nitrogen, and stored them at $-80^{\circ} \mathrm{C}$ until further processing. Protein extraction, quantification, digestion, and the following proteome profiling by 2D-LC-MS/MS (two-dimensional liquid chromatography tandem mass spectrometry) was done as described by Nestler et al. (2012) with multidimensional protein identification technology (MudPIT) analysis in three independent measurements that were combined to a total hit number of individual protein in each sample. Raw files were transformed to mascot generic format (mgf) and peptide hits were searched using the Open Mass Spectrometry Search Algorithm (OMSSA) against the Joint Genome Institute (JGI) Chlamydomonas protein sequence database Version 4.0 supplemented with 76 organelle encoded proteins (available online). ${ }^{8}$ False discovery rate for peptide hits was set to $1 \%$. Putative contamination by proteins from B. calyciflorus was determined with protein sequence data from Caenorhabditis elegans, another member of the Pseudocoelomata, because no sequence data was available for B. calyciflorus. Protein counts from three independent replicates of individual conditions were merged resulting in a final number of 2792 different proteins detected in at least one sample. Counts for individual proteins were normalized with the total number of protein counts for each sample and a Gtest, including a Benjamini-Hochberg correction, was used for further data analyses of differently expressed proteins. The $P$ value cutoffs for the detection of significantly different expression was set to 0.01 and a

${ }^{8}$ http://genome.jgi-psf.org/chlamy/chlamy.info.html false discovery rate of 0.05 was applied to account for multiple testing. Categorization of proteins to functional groups was performed based on the MapMan annotation (Thimm et al. 2004). The relative fraction of significantly up- or down-regulated proteins in each functional group was calculated by the ratio of significantly differently expressed proteins between conditions to the total number of proteins detected within this group in all samples.

Population dynamics and quantification of strain abundance with qPCR.-In order to determine how the phenotypic and genetic variation among algal strains can influence the overall dynamics of the predator-prey system, we performed a series of experimental trials of 35-d duration where we measured the dynamics of two algal strains and the rotifer population. We ran duplicate trials that included rotifers and all possible combinations of the two algal strains (for a total of 12 trials with rotifers), and additionally ran parallel trials in the absence of rotifers (six trials without rotifers). We used the trials without rotifers $(N=6)$ to confirm that the observed differences in growth rate between competing strains would lead to competitive exclusion by the fasting growing algal strain over the 35-d trial. We used the trials with rotifers $(N=12)$ in combination with models (see below) in order to test how different types of trait variation within algal populations affect the overall system dynamics.

All of our trials were conducted in $100-\mathrm{mL}$ cultures, and had a starting density of 1 rotifer $/ \mathrm{mL}$ (for the rotifer trials) and $10^{5}$ cells $/ \mathrm{mL}$ and for each algal strain in the mixture. Cultures were incubated in continuous light and diluted at a 1:5 ratio with fresh media every 2-3 days (i.e., $20 \%$ of the media was retained at each dilution step) such that overall dilution rate for each trial was $0.69 \mathrm{~d}^{-1}$. Rotifer population dynamics were quantified by counting live individuals after each dilution step. In order to ensure an accurate dilution rate of rotifers we took three replicate samples before dilution and then confirmed that the actual diluted sample fell within this range of expected post-diluted densities. Algal cell numbers were calculated after each dilution using fluorescence data by linear extrapolation with a stan- 
dard curve averaged from strain-specific fluorescence data.

We developed a qPCR method to quantify the dynamics of individual strains in the mixed algal populations over multiple generations. Algal cells were first separated from rotifers by filtration and subsequently washed with sterile water. DNA was extracted using a DNA extraction kit (Stratagene, La Jolla, California, USA) and analyzed with a Nanodrop ND1000 (NanoDrop Technologies, Wilmington, Delaware, USA). We used $300 \mathrm{ng}$ total genomic DNA for quantification of relative strain abundance in a nested PCR approach where the second reaction involved a quantitative real-time PCR (qPCR) reaction specific for each individual strain. Details about genomic sequences, amplification procedure and efficiency, strain specificity, and detection limits are described in the appendices (Appendix A: Table A1, Appendix C: Fig. C1).

Modeling of population dynamics. - We adapted previous models of the rotifer-algal system (Fussmann et al. 2000, Yoshida et al. 2003, Becks et al. 2010) so as to allow for trait differences between strains in terms of their growth rate, standing protection, and inducible defense. Population dynamics of the two-prey and onepredator strain over $35 \mathrm{~d}$ were fitted with a set of differential equations adapted from Becks et al. (2010). The model equations are

$$
\begin{aligned}
\frac{d C_{0}}{d t}= & q_{0} C_{0}\left(1-\frac{C_{0}+C_{1}}{k}\right)-\frac{\beta_{R}\left(1-\mathrm{s}_{0}\right) g_{0} C_{0} R}{\chi_{R}\left(K_{R}+\max \left\{Q^{*}, Q\right\}\right)} \\
& -\delta C_{0}
\end{aligned}
$$

$$
\begin{aligned}
\frac{d C_{1}}{d t}= & q_{1} C_{1}\left(1-\frac{C_{0}+C_{1}}{k}\right)-\frac{\beta_{R}\left(1-\mathrm{s}_{1}\right) g_{1} C_{1} R}{\chi_{R}\left(K_{R}+\max \left\{Q^{*}, Q\right\}\right)} \\
& -\delta C_{1}
\end{aligned}
$$

$$
\frac{d R}{d t}=\frac{\beta_{R} R Q}{K_{R}+\max \left\{Q^{*}, Q\right\}}-\delta R-m R
$$

where $C_{0}$ and $C_{1}$ are abundances of algae and $R$ is the abundance of rotifers, $g_{0}=\min \left\{1,(R / g)^{-i_{0}}\right\}$ and $g_{1}=$ $\min \left\{1,(R / g)^{-i_{1}}\right\}$ model the impact of inducible protection, and $Q=\left(1-\mathrm{s}_{0}\right) g_{0} C_{0}+\left(1-\mathrm{s}_{1}\right) g_{1} C_{1}$ is the prey availability taking into account both the standing and the induced defense (where $Q$ is the density of available and undefended prey [i.e., the edible algal cells of $C_{0}$ and $C_{1}$ ] and $Q^{*}$ is the critical prey density where the rotifer functional response switches from type-I to type-II [Becks et al. 2010]). The six variables $\left(s_{0}, s_{1}, q_{0}, q_{1}, i_{0}\right.$, and $i_{1}$ ) are the three trait values (for the two algal strains, $C_{0}$ and $C_{1}$, respectively), namely standing protection $(s)$, growth rate, $(q)$, and induced defense (i). Additional parameters are described in Table 2.
One of the assumptions of our modeling is that there is a continuous flux of nutrients and cells into and out of the system, as would be observed in a chemostat. Unlike many previous chemostat experiments that used similar models (Becks et al. 2012, 2010), we have performed our experiments in batch cultures with frequent dilutions to approximate a chemostat environment. To make our results more comparable to previous models and experiments we established a dilution rate of $0.69 \mathrm{~d}^{-1}$, which allowed us to maintain the populations for longer periods of time (e.g., 35 d) in order to potentially observe multiple predator-prey cycles.

To determine which trait differences between algal strains were necessary to best explain variation in the community dynamics, we compared the fit of eight model variants in which strains differed in up to three different trait values. Our simplest model variant is a purely ecological model because we forced both strains to have equal trait values. All other variants are ecoevolutionary models because we allowed differences in mean trait values between the two strains, subject to different combinations of the three constraints $\left(s_{0}=s_{1}\right.$, $q_{0}=q_{1}$, and $i_{0}=i_{1}$ ) that reflect different assumptions about the trait variability between strains. We fitted each of our model variants to all replicates of our rotifer trials $(N=12)$, and ranked the models using corrected Akaike information criterion $\left(\mathrm{AIC}_{\mathrm{c}}\right.$; Burnham and Anderson 2002). Fitting was performed on a parallel cluster using a Python implementation of the sequential least squares programming (SLSQP) constraint optimization algorithm (Ho and Harrington 2010, Flamholz et al. 2013), which finds the best fit of the model where the parameter space is restricted by pre-specified conditions, or constraints; in our case, a combination of the three parameter equalities $s_{0}=s_{1}$, $q_{0}=q_{1}$, and $i_{0}=i_{1}$ (Appendix B: Table B1). Using this approach, we optimized the log-likelihood of the model explaining the data

$$
\operatorname{LL}(\Theta \mid \hat{x})=\max _{\sigma} \sum_{i=1}^{n} \log \left(f\left(\hat{x}_{i} ; x_{i}(\Theta), \sigma^{2}\right)\right)
$$

where $\mathrm{LL}$ is log-likelihood, $\Theta=\left(s_{0}, s_{1}, q_{0}, q_{1}, i_{0}, i_{1}\right)$ is the vector of free parameters, $\hat{x}=\left(\hat{x}_{1}, \ldots \hat{x}_{n}\right)$ are the experimental data, $x(\Theta)=\left(x_{1}(\Theta), \ldots x_{n}(\Theta)\right)$ are the corresponding model predictions, and $f\left(\cdot ; \mu, \sigma^{2}\right)$ is the normal probability density function with mean $\mu$ and variance $\sigma^{2}$. For reasons of efficiency, we simulated the equations with a fixed time-step (i.e., as difference equations).

\section{RESUlts}

Phenotypic analysis.-We found significant differences among strains in their growth rate, standing protection, and induced defense (Table 1), and some evidence for variation in the strength of trade-offs between strains (Fig. 1). Initially we expected that fastergrowing strains would have lower levels of standing 

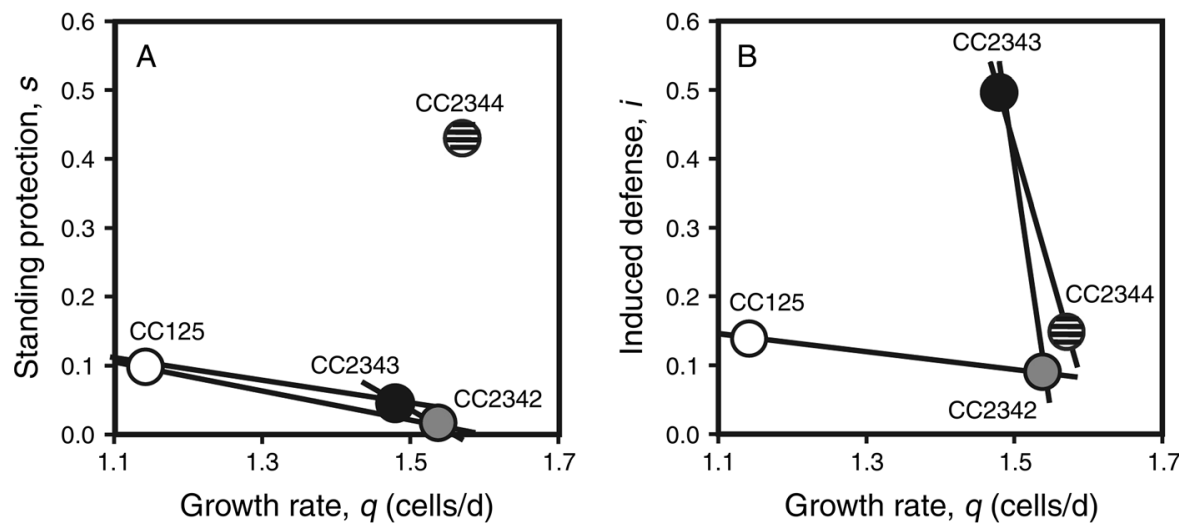

FIG. 1. Relationships between growth rate of four strains of Chlamydomonas reinhardtii and either standing protection in (A) the absence of rotifers or (B) induced defense in the presence of rotifers. Lines between the two strains indicate the potential for the presence of a trade-off between growth and defense.

protection, but this was not the case (Fig. 1A), as our fastest-growing strain (CC2344) also had the highest cell clumping ( $43 \%$ clumped) in the absence of predation (i.e., high standing protection). In addition, one of our strains strongly increased their cell clumping in the presence of predation (e.g., CC2343: from $5 \%$ to $62 \%$ ). Such an induced defense could increase its competitive ability against faster-growing strains (CC2342, CC2344) leading to potentially strong trade-offs between induced defense and growth (Fig. 1B).

The observed variation among strains in their induced defense traits means that changes in the genotype frequencies of algal strains over time could correspond to the evolution of reaction norms within the prey population. Previous work has found high values of broad-sense heritability for both growth rate and standing levels of protection (i.e., cell clumping) for different strains (Becks et al. 2010). In our study, trait differences among strains persisted over multiple generations when the strain was grown in the absence of rotifer predation (Table 1). This is consistent with previous work showing strain specificity of growth and standing protection traits (Becks et al. 2010), while additionally providing evidence for strain specificity of inducible defense traits.

To further investigate the genetic basis of defense traits and the phenotypic variability among our four algal strains we quantified protein expression profiles of each strain in the presence and absence of rotifers (Fig. 2A). From our analysis, we identified 2792 proteins to compare expression profiles among strains with no rotifer predation (Fig. 2A), and to compare within the same strain in the presence and absence of rotifers (Fig. 2B). These comparisons among short-term growth assays $(48 \mathrm{~h})$ revealed two important results. First, in the absence of rotifers the algal strains differed strongly in their protein expression across a broad range of cellular functions (Fig. 2A). This suggests a high level of genetic and phenotypic diversity among the strains, as expected from the contrasting growth and defense phenotypes of the strains (Table 1). Second, after $48 \mathrm{~h}$ of rotifers being present we observed strikingly similar responses of protein expression within certain functional groups of proteins (Fig. 2B). For example, rotifers caused all strains to up-regulate proteins involved in cellwall synthesis and general stress response, and, in addition, caused either up- or down-regulation of proteins involved in photosynthesis and protein synthesis (Fig. 2B). This suggests that rotifers can cause rapid changes to a wide range of proteins that could be associated with functional trait variation within algal populations and could be relevant for understanding the outcomes of predator-prey dynamics in our experimental system.

Competition experiments with and without predators.An important feature of our competition experiments is that we are assessing competitive differences between strains in terms of their maximum growth rate with unlimited nutrients, as opposed to competitive ability at limiting nutrient levels (Tilman et al. 1982). The rationale for this approach is that our frequent dilutions are replenishing nutrients sufficiently such that the fastest growing will most likely persist through time in the absence of rotifers. It is nevertheless possible that competitive interactions among our strains will change at different nutrient levels. For our experimental setup, where we conducted pairwise competition trials between algal strains without rotifers, we found that the fastergrowing strain nearly always became numerically dominant over the course of the 35-d trials (Fig. 3B, Appendix D: Fig. D1). The one exception to this was a case where the two algal strains had a very similar growth rate (CC2342 and CC2343; Fig. 3B). In trials with rotifers, we judged the outcome of competition based on changes in the relative abundance of cooccurring strains in the presence and absence of rotifers over the same timescale (i.e., from day 9-35; Fig. 3B).

Consistent with the large growth and defense trait variation among strains (Table 1), we found that the outcome of competition between strains in the presence 
of rotifers varied widely depending on the strain combination. For example, we found cases where predation seemed to (1) accelerate competitive exclusion (e.g., CC2344 vs. CC2342, CC2344 vs. CC125), (2) promote coexistence (CC125 vs. CC2342), (3) hinder coexistence by favoring the stronger-defended strain (CC2343 vs. CC2342), (4) have no effect (CC2343 vs. $\mathrm{CC} 125$ ), or (5) have contrasting effects among replicates (e.g., compare replicates of CC2344 vs. CC2343; Fig. 3B). This latter effect could be explained if tradeoffs between growth and defense vary in either their occurrence or strength in relation to rotifer density. This appeared to be the case for the two replicate trials with the strain that has the fastest growth rate and a high level of standing defense (CC2344) and the strain with a fast growth rate and the greatest induced defense (CC2343; Fig. 3C).

Overall, such algal dynamics in these experiments confirm that eco-evolutionary dynamics are potentially important, because strains with heritable trait variation are changing in their frequency over time, and the specific combination of strains seems to qualitatively affect overall system dynamics (Appendix D: Fig. D2). In order to quantitatively test which specific trait differences among algal prey were the most important for explaining overall community dynamics we compared the fit of a suite of eco-evolutionary models to the observed dynamics.

Comparison of eco-evolutionary models.-Our purely ecological model, which allowed for plasticity in defense but not for variation in plasticity between strains (i.e., no intrapopulation variation in reaction norms), was never a likely explanation for the observed community dynamics (Fig. 4, Appendix B: Table B1). Instead, the most common best-fitting model, which is an ecoevolutionary variant, allowed for differences between strains in their inducibility of defense (Fig. 4). This model variant performed particularly well when the competing algal strains differed markedly in inducible defense trait (Table 1); for example, when CC2343 was present (Fig. 4, Appendix B: Table B1). By comparison, eco-evolutionary model variants where only standing protection differed between strains fitted well to competition trials where the strain with the highest standing levels of protection was present (i.e., CC2344). More generally, identifying the best-fitting models depends on the specific combinations of strains present in the system and the size of the trait differences between strains. Across all experiments, the fitted model parameters matched well with independently measured trait values (Appendix E: Fig. E1), providing additional support for our models and fitting procedure.

\section{DisCUSSION}

Although much is known about the evolution of reaction norms (Stearns and Koella 1986, Thompson 1991, Scheiner 1993), research on the rapid evolution of phenotypic plasticity is currently poorly integrated into studies on eco-evolutionary dynamics (Cortez 2011, Ellner et al. 2011, Vasseur et al. 2011, Hanski 2012). There is ample evidence for genetic variation of plastic traits in natural populations (Via et al. 1995, WestEberhard 2003, Pigliucci 2005), and both compelling theory and empirical examples demonstrating how such plastic traits can evolve in response to selection (Via and Lande 1985, Stearns and Koella 1986, Brakefield et al. 1996, West-Eberhard 2005). There are numerous examples in natural populations where plasticity could evolve relatively rapidly; for example, from de novo mutations during development (Brakefield et al. 1996), from standing genetic variation underlying phenotypically plastic traits (Tollrian 1995), and from selection on reaction norms (Olsen et al. 2004). The presence of heritable variation in the phenotypic response of individuals to a range of environmental conditions (Stearns and Koella 1986, Pigliucci 2005) opens up the possibility that for a particular eco-evolutionary scenario the evolving trait could be the reaction norm of a phenotypically plastic trait. Indeed, our experiments show that we can add significant explanatory power to the fit of eco-evolutionary models if we additionally consider intrapopulation variation of prey reaction norms.

In our experiments, genetically different prey strains varied in their induced responses to predation, both at the level of morphological changes associated with palmella formation (Table 1) and at the level of protein expression (Fig. 2). For example, strain CC2344 had the highest palmella formation in the absence of rotifers (Table 1) and significantly overexpressed more proteins involved in cell-wall synthesis compared to all other strains (Fig. 2A). In the presence of rotifers, this strain also dramatically increased levels of protein expression across a broad range of functions (green line in Fig. 2B). More generally, we found some striking similarities in the proteomic responses of our algal strains to the presence of rotifers (Fig. 2B), as all four strains significantly up-regulated the expression of proteins associated with both cell wall synthesis and stress response (Fig. 2B). If proteins involved in cell-wall synthesis are associated with palmella formation during predation stress (Becks et al. 2012), this would support the notion that palmella induction has a genetic basis and that its expression in response to rotifer density varies among strains over short time periods. Although we are a long way off from explaining the direct link between variation in protein expression and the traits governing community dynamics, our proteomic analyses reveal clear strain-specific differences in expression patterns across multiple functional groups of proteins (Fig. 2B). This is consistent with there being variation in plasticity among strains that is expressed in the presence of rotifers over short time periods relative to the observed predator-prey dynamics.

Variation in both growth and defense traits of our experimentally assembled algal populations had signif- 


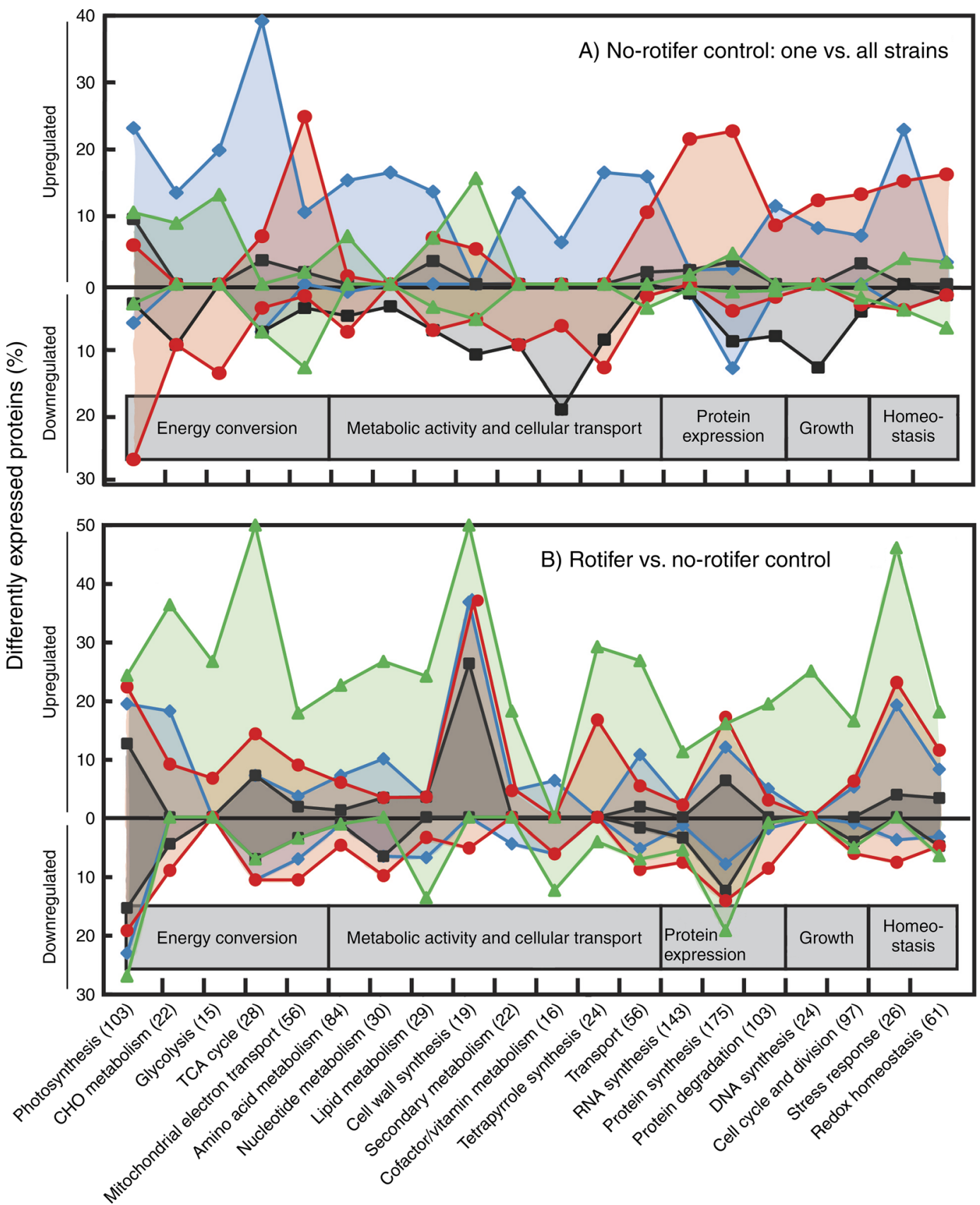

FIG. 2. Comparison of protein expression profiles of the strains CC125 (blue diamonds), CC2342 (black squares), CC2343 (red circles), and CC2344 (green triangles) grown either in the absence (no-rotifer control) or the presence of rotifers for $48 \mathrm{~h}$. Variation in the percentage of significantly different classes of expressed proteins (up- or down-regulation) for all detected proteins (number in parentheses) were analyzed for various functional groups based on MapMan annotation (Thimm et al. 2004) by comparing either (A) protein expression of one strain relative to all other strains, all in the absence of rotifers (among strain analysis), or (B) the protein expression of each strain in the presence and absence of rotifers (within strain analysis). Classes of proteins are shown on the $x$-axis and numbers in parentheses are the number of individual proteins in that class. The names of all the proteins in each class are summarized in the Supplement (AnnotatedProteomics.csv).

icant impacts on overall community dynamics in general, and on the outcome of competition between prey strains in particular. In the absence of predation, we found that the faster-growing strain always outcompeted the slower-growing strain over the 35-d trials
(Appendix D: Fig. D1) and that greater numerical dominance was explained by bigger differences in growth rate between strains independent of variation in defense traits (Fig. 3B). In the presence of rotifers, the outcome of competition between strains was more 

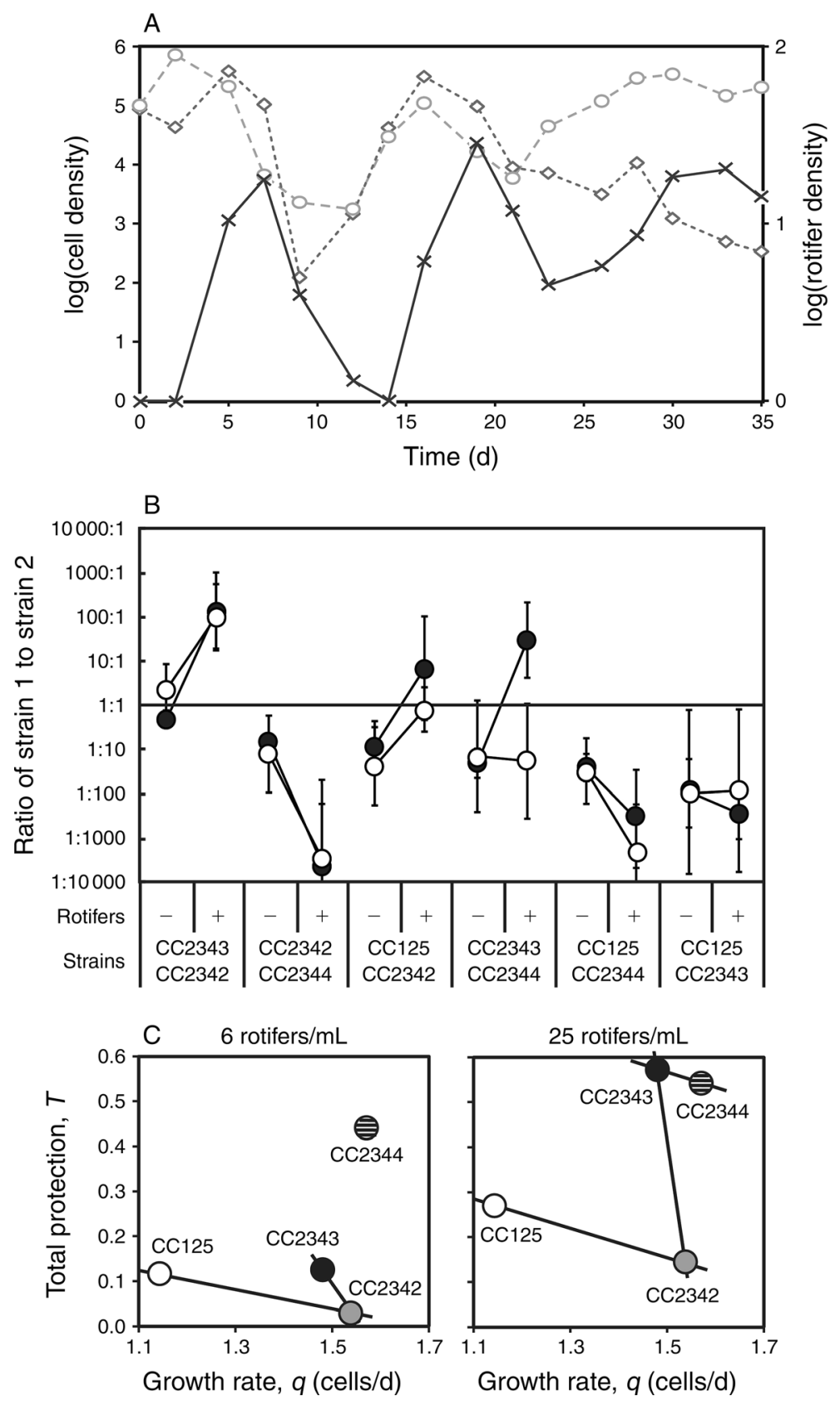

FIG. 3. (A) Representative graph of the population dynamics over $35 \mathrm{~d}$ of a pairwise competition trial of algae (dashed and dotted lines) in the presence of rotifers (solid line). Strain CC125 (circles, dashed line) and strain CC2342 (diamonds, dotted line) were initially mixed in a 1:1 ratio to a total density of $2 \times 10^{5}$ cells $/ \mathrm{mL}$ with a rotifer density of 1 individuals $/ \mathrm{mL}$. (B) The average ratio of strain 1 (top, slower growing) to strain 2 (bottom, faster growing) during day 9-35 for all strain combinations either in the absence $(-)$ or presence $(+)$ of rotifers in two replicates (solid and open circles for replicates 1 and 2, respectively). Strain combinations are ordered from smallest difference in growth (left) to largest difference in growth (right). (C) Trade-offs (black lines) between growth $(q)$ and total defense $(s+i(R))$ of different strains at two different rotifer densities $(R)$.

variable, and seemed to depend on trade-offs between growth and defense traits (Fig. 1). In one instance, for example, rotifer predation allowed a slow-growing strain with a strong defense (e.g., CC125) to better compete with a fast-growing but less well-defended clone (e.g., CC2342; Fig. 3B). In other instances, where differences in growth rate between strains were small, we found that the most competitive clone in the presence of rotifers either had a higher level of standing protection (CC2344 vs. CC2342) or a greater induction of defense (CC2343 vs. CC2342). In yet another instance, the outcome of competition between two trials (CC2343 vs. CC2344) depended on how growth-defense trade-offs between strains varied with rotifer density (Fig. 3C). For 
FIG. 4. Summary of the number of best fits (i.e., the change in Akaike's information criterion corrected for small sample sizes $\left.\left[\Delta \mathrm{AIC}_{\mathrm{c}}\right]=0\right)$ and good fits $\left(\Delta \mathrm{AIC}_{\mathrm{c}}<2\right)$ for the ecological model and eco-evolutionary models of varying complexity. The fits were determined for each pairwise competition trial $(N=12)$ and based on a range of models with different constraints on the parameters ( $i$, induced defense; $s$, standing protection; and $q$, growth rate). An $\mathrm{X}$ indicates that the parameter was allowed to differ between strains during the model fitting. The strain combinations for each replicate are indicated by half circles with different fill (CC125, white; CC2342, gray; CC2343, black; CC2344, striped), and the star indicates a tie for the best-fitting model (Appendix B: Table B1).

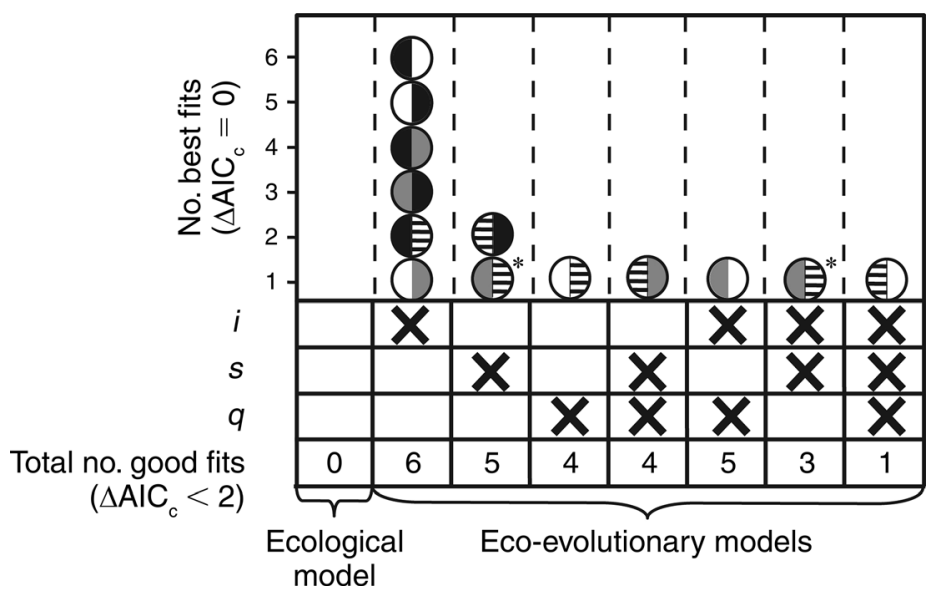

example, strain CC2343 only outcompeted CC2344 at high rotifer densities, where a potential trade-off could emerge between total defense and growth rate (Fig. 3C). Although we are unable to test the repeatability of such outcomes with only two replicates for each pairwise trial, our results are consistent with their being intrapopulation variation in defense traits that have functional consequences for predicting overall system dynamics.

Previous research in this rotifer-algal system has clearly demonstrated the interplay between the ecological and evolutionary effects that constitute eco-evolutionary feedbacks (Becks et al. 2010, 2012). The initial evolutionary effect occurs because rotifers drive an increase in the frequency of well-defended algal genotypes through time. Such predator-mediated prey evolution has the ecological effect of lowering predator population density and allowing prey populations to subsequently increase. An eco-evolutionary feedback occurs because prey evolution is subsequently mediated by competition for resources in a low-predation environment, eventually leading to prey genotypes with faster growth rates and poorer defenses. Many of the previous experiments in this system were run in chemostats with a fixed nutrient supply that allowed for intense competition for resources at low rotifer densities (Becks et al. 2010, 2012). However, we ran our experiments in batch cultures that were diluted every few days, meaning that competition for resources may have been less intense. This does not preclude the possibility of eco-evolutionary dynamics. In the absence of rotifers, the fastest growing strains became numerically dominant over the course of the experiment, and in the presence of rotifers there was a clear effect of predation on the frequencies of different algal strains through time (i.e., phenotypic evolution of the algal population). By using different prey genotypes we could manipulate the standing levels of intrapopulation variation in the growth and defense traits of prey (Table 1), so as to test which phenotypic differences are significant predictors of overall community dynamics. Our observed dynamics (Fig. 3B, Appendix D: Fig. D2) suggest that combinations of prey strains with different growth rates, standing levels of protection, and induced defenses (evolutionary factors) qualitatively affected predatorprey population dynamics (ecological factors). In order to quantitatively confirm this, we tested the fit of our observed community dynamics to a range of ecoevolutionary models (Fig. 4).

The poor fit of our ecological model to all of our experimental trials confirms previous work in this system showing that the mere presence of phenotypic plasticity is unlikely to explain the observed algal-rotifer community dynamics (Fussmann et al. 2000, Yoshida et al. 2003, 2004, Becks et al. 2010, 2012). Indeed, it is the genetic differences among algal strains underlying growth-defense trade-offs that strongly influences the outcome of predator-prey dynamics (Yoshida et al. 2003, Becks et al. 2010). This is also supported by the observation that multiple strains isolated from a mixed algal community exhibited strong heritability of the clumping phenotypes (Becks et al. 2010). However, we additionally find that the best-fitting eco-evolutionary model allows for intrapopulation variation in inducible defense traits between prey genotypes (Fig. 4). This suggests that intrapopulation variation in the reaction norms of plastic traits (i.e., induced defense) can be an important component of eco-evolutionary dynamics (Chevin et al. 2012, Cameron et al. 2013), and that trade-offs among such traits can influence both the likelihood of coexistence and overall community dynamics.

Overall, our results contribute to multiple, but poorly integrated, lines of research within the broad field of eco-evolutionary dynamics. For example, we provide additional evidence that (1) rapid evolution occurs over timescales that are relevant for understanding ecological dynamics (Hairston et al. 2005, Ellner et al. 2011), (2) genetic variation of primary producers can strongly affect not only the structure (Crutsinger et al. 2006, Johnson 2010) but also the dynamics of upper trophic levels (Keith et al. 2010, Whitham et al. 2012), and that 
(3) variation in phenotypic plasticity can underlie the dynamics of interacting populations (Coulson et al. 1998, 2006, Pelletier et al. 2007, Cameron et al. 2013). Related to this last point, our work highlights the importance of considering the combined effects of phenotypic plasticity and genetic diversity on community dynamics (Yamamichi et al. 2011, Kovach-Orr and Fussmann 2012). There is ample evidence for genetic variation of plastic traits in natural populations (Via et al. 1995, West-Eberhard 2003, Pigliucci 2005), and both compelling theory and empirical examples demonstrating how such traits can evolve in response to selection in general (Via and Lande 1985, Stearns and Koella 1986, Brakefield et al. 1996, West-Eberhard 2005), and to predator-mediated selection in particular (Arnqvist and Johansson 1998, Beldade and Brakefield 2002, Relyea 2003). Our work suggests that variation in plastic traits could underlie the phenotypic changes associated with certain eco-evolutionary dynamics (Hanski 2012), and highlights the need for more comparative and experimental work exploring how trait variation that is either genetically or environmentally induced can influence eco-evolutionary dynamics in natural populations.

\section{ACKNOWLEDGMENTS}

We thank René Schönenberger, Noemi Mariacher, Ksenia Groh, and Holger Nestler for their technical and scientific assistance, and Luke Harmon for advice on the model fitting. We thank Nelson Hairston and Jonathan Levine for thoughtful comments on the manuscript. We thank the Eawag Directorate for funding.

\section{Literature Cited}

Agrawal, A., M. Johnson, A. Hastings, and J. Maron. 2013. A field experiment demonstrating plant life-history evolution and its eco-evolutionary feedback to seed predator populations. American Naturalist 181:S35-S45.

Arnqvist, G., and F. Johansson. 1998. Ontogenetic reaction norms of predator-induced defensive morphology in dragonfly larvae. Ecology 79:1847-1858.

Bassar, R. D., R. Ferriere, A. López-Sepulcre, M. C. Marshall, J. Travis, C. M. Pringle, and D. N. Reznick. 2012. Direct and indirect ecosystem effects of evolutionary adaptation in the Trinidadian guppy (Poecilia reticulata). American Naturalist 180:167-185.

Becks, L., S. P. Ellner, L. E. Jones, and N. G. Hairston. 2010. Reduction of adaptive genetic diversity radically alters ecoevolutionary community dynamics. Ecology Letters 13:989997.

Becks, L., S. P. Ellner, L. E. Jones, and N. G. Hairston, Jr. 2012. The functional genomics of an eco-evolutionary feedback loop: linking gene expression, trait evolution, and community dynamics. Ecology Letters 15:492-501.

Beldade, P., and P. M. Brakefield. 2002. The genetics and evodevo of butterfly wing patterns. Nature Reviews Genetics 3: $442-452$.

Bolnick, D. I., P. Amarasekare, M. S. Araujo, R. Bürger, J. M. Levine, M. Novak, V. H. W. Rudolf, S. J. Schreiber, M. C. Urban, and D. A. Vasseur. 2011. Why intraspecific trait variation matters in community ecology. Trends in Ecology and Evolution 26:183-192.

Brakefield, P. M., J. Gates, D. Keys, F. Kesbeke, P. J. Wijngaarden, A. Monteiro, V. French, and S. B. Carroll. 1996. Development, plasticity and evolution of butterfly eyespot patterns. Nature 384:236-242.
Burnham, K. P., and D. R. Anderson. 2002. Model selection and multimodel inference: a practical information-theoretic approach. Second edition. Springer-Verlag, New York, New York, USA.

Cameron, T. C., D. O'Sullivan, A. Reynolds, S. B. Piertney, and T. G. Benton. 2013. Eco-evolutionary dynamics in response to selection on life-history. Ecology Letters 16:754763.

Chevin, L.-M., S. Collins, and F. Lefèvre. 2012. Phenotypic plasticity and evolutionary demographic responses to climate change: taking theory out to the field. Functional Ecology 1: $1-12$.

Cortez, M. H. 2011. Comparing the qualitatively different effects rapidly evolving and rapidly induced defences have on predator-prey interactions. Ecology Letters 14:202-209.

Coulson, T. N., S. D. Albon, J. M. Pemberton, J. Slate, F. E. Guinness, and T. H. Clutton-Brock. 1998. Genotype by environment interactions in winter survival in red deer. Journal of Animal Ecology 67:434-445.

Coulson, T., T. G. Benton, P. Lundberg, S. Dall, and B. E. Kendall. 2006. Putting evolutionary biology back in the ecological theatre: a demographic framework mapping genes to communities. Evolutionary Ecology Research 8:11551171.

Crutsinger, G. M., M. D. Collins, J. A. Fordyce, Z. Gompert, C. C. Nice, and N. J. Sanders. 2006. Plant genotypic diversity predicts community structure and governs an ecosystem process. Science 313:966-968.

Ellner, S. P., M. A. Geber, and J. N. G. Hairston. 2011. Does rapid evolution matter? Measuring the rate of contemporary evolution and its impacts on ecological dynamics. Ecology Letters 14:603-614.

Ezard, T. H. G., S. D. Cote, and F. Pelletier. 2009. Ecoevolutionary dynamics: disentangling phenotypic, environmental and population fluctuations. Philosophical Transactions of the Royal Society B 364:1491-1498.

Farkas, T. E., T. Mononen, A. A. Comeault, I. Hanski, and P. Nosil. 2013. Evolution of camouflage drives rapid ecological change in an insect community. Current Biology 23:18351843.

Fischer, B. B., S. Roffler, and R. I. L. Eggen. 2012. Multiple stressor effects of predation by rotifers and herbicide pollution on different Chlamydomonas strains and potential impacts on population dynamics. Environmental Toxicology and Chemistry 31:2832-2840.

Flamholz, A., E. Noor, A. Bar-Even, W. Liebermeister, and R. Milo. 2013. Glycolytic strategy as a tradeoff between energy yield and protein cost. Proceedings of the National Academy of Sciences USA 110:10039-10044.

Fussmann, G. F., S. P. Ellner, K. W. Shertzer, and N. G. Hairston. 2000. Crossing the hopf bifurcation in a live predator-prey system. Science 290:1358-1360.

Fussmann, G. F., M. Loreau, and P. A. Abrams. 2007. Ecoevolutionary dynamics of communities and ecosystems. Functional Ecology 21:465-477.

Hairston, J. N. G., S. P. Ellner, M. A. Geber, T. Yoshida, and J. A. Fox. 2005. Rapid evolution and the convergence of ecological and evolutionary time. Ecology Letters 8:11141127.

Hanski, I. 2012. Eco-evolutionary dynamics in a changing world. Annals of the New York Academy of Sciences 1249:117.

Harmon, L. J., B. Matthews, S. Des Roches, J. M. Chase, J. B. Shurin, and D. Schluter. 2009. Evolutionary diversification in stickleback affects ecosystem functioning. Nature 458:11671170.

Ho, K., and H. A. Harrington. 2010. Bistability in apoptosis by receptor clustering. PLoS Computational Biology 6: e1000956. 
Johnson, M. T. J. 2010. The contribution of evening primrose (Oenothera biennis) to a modern synthesis of evolutionary ecology. Population Ecology 53:9-21.

Keith, A. R., J. K. Bailey, and T. G. Whitham. 2010. A genetic basis to community repeatability and stability. Ecology 91 : 3398-3406.

Kokko, H., and A. López-Sepulcre. 2007. The ecogenetic link between demography and evolution: can we bridge the gap between theory and data? Ecology Letters 10:773-782.

Kovach-Orr, C., and G. F. Fussmann. 2012. Evolutionary and plastic rescue in multitrophic model communities. Philosophical Transactions of the Royal Society B 368:20120084.

Lurling, M., and W. Beekman. 2006. Palmelloids formation in Chlamydomonas reinhardtii: defence against rotifer predators? Annales de Limnologie 42:65-72.

Martínez-Fernández, M., M. de la Cadena, and E. RolánAlvarez. 2010. The role of phenotypic plasticity on the proteome differences between two sympatric marine snail ecotypes adapted to distinct micro-habitats. BMC Evolutionary Biology 10:65.

Matthews, B., et. al. 2011. Toward an integration of evolutionary biology and ecosystem science. Ecology Letters 14:690-701.

Nestler, H., K. J. Groh, R. Schönenberger, R. I. L. Eggen, and M. J. F. Suter. 2012. Linking proteome responses with physiological and biochemical effects in herbicide-exposed Chlamydomonas reinhardtii. Journal of Proteomics 75:53705385.

Olsen, E. M., M. Heino, G. R. Lilly, M. J. Morgan, J. Brattey, B. Ernande, and U. Dieckmann. 2004. Maturation trends indicative of rapid evolution preceded the collapse of northern cod. Nature 428:932-935.

Ozgul, A., D. Z. Childs, M. K. Oli, K. B. Armitage, D. T. Blumstein, L. E. Olson, S. Tuljapurkar, and T. Coulson. 2010. Coupled dynamics of body mass and population growth in response to environmental change. Nature 466: $482-485$.

Ozgul, A., S. Tuljapurkar, T. G. Benton, J. M. Pemberton, T. H. Clutton-Brock, and T. Coulson. 2009. The dynamics of phenotypic change and the shrinking sheep of St. Kilda. Science 325:464-467.

Pelletier, F., T. Clutton-Brock, J. Pemberton, S. Tuljapurkar, and T. Coulson. 2007. The evolutionary demography of ecological change: linking trait variation and population growth. Science 315:1571-1574.

Pigliucci, M. 2005. Evolution of phenotypic plasticity: where are we going now? Trends in Ecology and Evolution 20:481486.

Post, D. M., and E. P. Palkovacs. 2009. Eco-evolutionary feedbacks in community and ecosystem ecology: interactions between the ecological theatre and the evolutionary play. Philosophical Transactions of the Royal Society B 364:16291640.

Relyea, R. A. 2003. How prey respond to combined predators: a review and an empirical test. Ecology 84:1827-1839.

Reznick, D. N. 2013. A critical look at reciprocity in ecology and evolution: introduction to the symposium. American Naturalist 181:S1-S8.

Scheiner, S. M. 1993. Genetics and evolution of phenotypic plasticity. Annual Review of Ecology and Systematics 24:3568.

Schoener, T. W. 2011. The newest synthesis: understanding the interplay of evolutionary and ecological dynamics. Science 331:426-429.

Schreiber, S., R. Burger, and D. Bolnick. 2011. The community effects of phenotypic and genetic variation within a predator population. Ecology 92:1582-1593.

Silvestre, F., V. Gillardin, and J. Dorts. 2012. Proteomics to assess the role of phenotypic plasticity in aquatic organisms exposed to pollution and global warming. Integrative and Comparative Biology 52:681-694.

Smallegange, I. M., and T. Coulson. 2013. Towards a general, population-level understanding of eco-evolutionary change. Trends in Ecology and Evolution 28:143-148.

Stearns, S. C., and J. C. Koella. 1986. The evolution of phenotypic plasticity in life-history traits: predictions of reaction norms for age and size at maturity. Evolution 40: 893-913.

Thimm, O., O. Bläsing, Y. Gibon, A. Nagel, S. Meyer, P. Krüger, J. Selbig, L. A. Müller, S. Y. Rhee, and M. Stitt. 2004. MAPMAN: a user-driven tool to display genomics data sets onto diagrams of metabolic pathways and other biological processes. Plant Journal 37:914-939.

Thompson, J. D. 1991. Phenotypic plasticity as a component of evolutionary change. Trends in Ecology and Evolution 6: 246-249.

Thompson, J. N. 1998. Rapid evolution as an ecological process. Trends in Ecology and Evolution 13:329-332.

Tilman, D., S. Kilham, and P. Kilham. 1982. Phytoplankton community ecology: the role of limiting nutrients. Annual Review of Ecology and Systematics 13:349-372.

Tollrian, R. 1995. Predator-induced morphological defenses: costs, life history shifts, and maternal effects in Daphnia pulex. Ecology 76:1691-1705.

Van der Stap, I., M. Vos, B. W. Kooi, B. T. M. Mulling, E. van Donk, and W. M. Mooij. 2009. Algal defenses, population stability, and the risk of herbivore extinctions: a chemostat model and experiment. Ecological Research 24:1145-1153.

Van der Stap, I., M. Vos, and W. M. Mooij. 2006. Linking herbivore-induced defences to population dynamics. Freshwater Biology 51:424-434.

Van der Stap, I., M. Vos, and W. M. Mooij. 2007. Inducible defenses and rotifer food chain dynamics. Hydrobiologia 593:103-110.

Vasseur, D. A., P. Amarasekare, V. H. W. Rudolf, and J. M. Levine. 2011. Eco-evolutionary dynamics enable coexistence via neighbor-dependent selection. American Naturalist 178: E96-E109.

Verschoor, A. M., M. Vos, and I. van der Stap. 2004. Inducible defences prevent strong population fluctuations in bi- and tritrophic food chains. Ecology Letters 7:1143-1148.

Via, S., R. Gomulkiewicz, G. DeJong, S. M. Scheiner, C. D. Schlichting, and P. H. van Tienderen. 1995. Adaptive phenotypic plasticity: consensus and controversy. Trends in Ecology and Evolution 10:212-217.

Via, S., and R. Lande. 1985. Genotype-environment interaction and the evolution of phenotypic plasticity. Evolution 39:505522 .

Vos, M., B. W. Kooi, D. L. DeAngelis, and W. M. Mooij. 2004. Inducible defences and the paradox of enrichment. Oikos 105:471-480.

West-Eberhard, M. J. 2003. Developmental plasticity and evolution. Oxford University Press, New York, New York, USA.

West-Eberhard, M. J. 2005. Developmental plasticity and the origin of species differences. Proceedings of the National Academy of Sciences USA 102:6543-6549.

Whitham, T. G., C. A. Gehring, L. J. Lamit, T. Wojtowicz, L. M. Evans, A. R. Keith, and D. S. Smith. 2012. Community specificity: life and afterlife effects of genes. Trends in Plant Science 17:271-281.

Yamaguchi, W., M. Kondoh, and M. Kawata. 2011. Effects of evolutionary changes in prey use on the relationship between food web complexity and stability. Population Ecology 53: $59-72$.

Yamamichi, M., T. Yoshida, and A. Sasaki. 2011. Comparing the effects of rapid evolution and phenotypic plasticity on predator-prey dynamics. American Naturalist 178:287-304. 
Yoshida, T., S. P. Ellner, L. E. Jones, and B. Bohannan. 2007. Cryptic population dynamics: rapid evolution masks trophic interactions. PLoS Biology 5:1868-1879.

Yoshida, T., N. G. Hairston, and S. P. Ellner. 2004. Evolutionary trade-off between defence against grazing and competitive ability in a simple unicellular alga, Chlorella vulgaris. Proceedings of the Royal Society B 271:1947-1953. Yoshida, T., L. E. Jones, S. P. Ellner, G. F. Fussmann, and N. G. Hairston. 2003. Rapid evolution drives ecological dynamics in a predator-prey system. Nature 424:303-306.

Supplemental Material

\section{Ecological Archives}

Appendices A-E and the Supplement are available online: http://dx.doi.org/10.1890/14-0116.1.sm 